\title{
PERFIL AUDIOMÉTRICO DE MÚSICOS PROFISSIONAIS: REVISÃO SISTEMÁTICA
}

\section{Audiometric profile of professional musicians: systematic review \\ Perfil audiometrico de músicos profesionales: revisión integrativa}

\author{
Carina Moreno Dias Carneiro Muniz \\ Universidade CEUMA - CEUMA - São Luis (MA) - Brasil \\ Camila Malcher Teixeira Amorim \\ Universidade CEUMA - CEUMA - São Luis (MA) - Brasil \\ Ilana Mirian Almeida Felipe \\ Universidade CEUMA - CEUMA - São Luis (MA) - Brasil \\ Rosane da Silva Dias \\ Universidade CEUMA - CEUMA - São Luis (MA) - Brasil
}

\section{RESUMO}

Objetivo: Conhecer o perfil audiológico de músicos com dedicação profissional. Métodos: Conduziu-se uma revisão sistemática da literatura nas bases eletrônicas Periódicos CAPES e Biblioteca Virtual em Saúde (BVS) utilizando os descritores "música", "perda auditiva induzida pelo ruído", "zumbido" e "audiometria". Na base PubMed, utilizaram-se os termos MESH: "music", "audiometry" e "hearing loss", utilizando os operadores booleanos "e" e "and". Incluíram-se estudos observacionais, em inglês, espanhol e português, que avaliaram a ocorrência e os fatores relacionados à perda auditiva em músicos profissionais, datados de 2006 a 2016. A seleção dos artigos aconteceu por pares. Resultados: A estratégia de busca resultou em 819 estudos, dos quais apenas 13 preencheram os critérios de elegibilidade. Dos músicos avaliados pelos treze estudos incluídos, $31,24 \%$ apresentaram perda auditiva induzida pelo ruído. Conclusão: Por meio dessa revisão foi possível identificar que os achados da literatura são consensuais quanto à detecção de que músicos apresentam perda auditiva induzida por ruído, sendo o zumbido o sintoma auditivo mais frequente, o que é indicativo que existe alteração na audição desses profissionais.

Descritores: Música; Audiometria; Perda Auditiva Provocada por Ruído; Ruído Ocupacional.

\section{ABSTRACT}

Objective: To know professional musicians'audiometric profile. Methods: A systematic review of the literature was carried out in the online databases of CAPES Journals and Virtual Health Library (VHL) using the descriptors "music", "noise-induced hearing loss", "tinnitus" and "audiometry". In the PubMed database, we used the MESH headings: "music", "audiometry" and "hearing loss", using Boolean term "and". We included observational studies in English, Spanish and Portuguese that assessed the occurrence and factors related to hearing loss in professional musicians dating from 2006 to 2016. The articles were peer-selcted. Results: The searching strategy resulted in 819 studies, of which only 13 met the eligibility criteria. Of all the musicians analyzed by the thirteen studies included in this review, 31,24\% presented noise-induced hearing loss. Conclusion: The present review verified a consensus among the findings of the studies analyzed regarding the fact that musicians present noise-induced hearing loss, with tinnitus being the most frequent symptom, which indicates the existence of hearing alterations among these professionals.

Descriptors: Music; Audiometry; Hearing Loss, Noise-Induced; Noise, Occupational. 


\section{RESUMEN}

Objetivo: Conocer el perfil audiológico de músicos con dedicación profesional. Métodos: Se realizó una revisión sistemática de la literatura en las bases de datos electrónicas Periódicos CAPES y Biblioteca Virtual en Salud (BVS) utilizando los descriptores "música", "pérdida auditiva provocada por el ruido", "zumbido" y "audiometría". En la base PubMed se utilizaron los términos MESH: "music", "audiometry" $y$ "hearing loss" con los operadores booleanos " $e$ ” $y$ "and". Se incluyeron los estudios observacionales, en inglés, español y portugués que evaluaron la ocurrencia y los factores relacionados con la pérdida auditiva de músicos profesionales entre 2006 y 2016. La selección de los artículos se dio por pares. Resultados: La estrategia de búsqueda resultó en 819 estudios de los cuales solamente 13 cumplieron los criterios de elegibilidad. De entre los músicos evaluados en los trece estudios incluidos, el 31,24\% presentaron pérdida auditiva provocada por el ruido. Conclusión: A partir de esa revisión ha sido posible identificar que los hallazgos de la literatura presentan un consenso respecto la detección de que los músicos presentan pérdida auditiva provocada por el ruido siendo el zumbido el síntoma auditivo más frecuente lo que es una indicación de que hay alteración de la audición de estos profesionales.

Descriptores: Música; Audiometría; Pérdida Auditiva Provocada por Ruido; Ruido en el Ambiente de Trabajo.

\section{INTRODUÇÃO}

As pessoas nascem com a audição normal, porém há um número significativo delas que apresenta perda auditiva. Dentre as causas mais comuns estão as de ordem congênita, a exemplo da rubéola e da sífilis, e as adquiridas, grupo em que se incluem aqueles que estão continuamente expostos a ruído excessivo, especialmente o ruído ocupacional, que tem-se mostrado com características de perda auditiva irreversível $^{(1,2)}$.

Segundo a Organização Mundial de Saúde (OMS), mais de 5\% da população mundial, cerca de 360 milhões de pessoas, têm surdez incapacitante (328 milhões de adultos e 32 milhões de crianças). Grande parte desses vivem em países subdesenvolvidos ${ }^{(3)}$. Estima-se que 1,1\% da população brasileira se declara incapaz, com dificuldade permanente para ouvir ${ }^{(4)}$.

É crescente o número de casos de perda auditiva em pessoas expostas a ruídos ocupacionais. Nesse caso, o ambiente laboral tem sido gerador de ruído elevado e a falta de proteção adequada não é incomum ${ }^{(5)}$. Os músicos estão sob risco considerável de dano auditivo por estarem regularmente expostos a níveis elevados de pressão sonora em razão da fonte destes sons estar muito próxima de seus ouvidos. Além disso, há resistência por parte dos músicos ao uso de protetores auriculares ${ }^{(6)}$. Um estudo conduzido com músicos de Rock, na Noruega, encontrou 37,8\% dos exames audiômetros configurados em entalhe, compatíveis com perda auditiva induzida pelo ruído (PAIR) ${ }^{(7)}$.

Ressalte-se que os profissionais da música não contam com amparo legal, no que se refere à saúde e à segurança no trabalho, o que pode remeter a uma ideia errônea de que ser músico não é ser trabalhador, ou de que, se a música for agradável, não gera risco à saúde ${ }^{(8)}$. Na mesma linha, estudos reiteram prejuízos ocupacionais entre os músicos, entre eles estão os danos $\operatorname{auditivos}^{(9,10)}$.

São escassos estudos que investiguem os impactos do ruído na audição de músicos ou que apontem, de forma fundamentada, os fatores que a afetam. Nessa direção, por considerar que a habilidade auditiva é um recurso importante para esse profissional, assim como para sua qualidade de vida, pesquisas que visem compreender os efeitos do ruído na audição desses profissionais tornam-se relevantes. Com base nesse contexto, a presente revisão objetivou conhecer o perfil audiológico de músicos com dedicação profissional.

\section{MÉTODOS}

Para cumprir com o objetivo proposto, realizou-se uma revisão sistemática da literatura com fundamento nas diretrizes determinadas pelo Preferred Reporting Items for Systematic Reviews and Meta-Analyses - PRISMA(11). No processo de coleta, a questão problema foi organizada a partir da estratégia PICO, sendo "P" para patients or population/paciente ou população, "I" para intervention or area of interest/intervenção ou fator de exposição, "C" para comparison/grupo controle e “O” para outcomes/desfecho. Assim resultou: P: Músicos profissionais; I: exposição à música em rotina profissional; C: grupo comparativo/controle; O: critério diagnóstico para perda auditiva realizada por audiometria ou emissões otoacústicas.

Dessa maneira, ficou definida a seguinte questão norteadora da pesquisa: uma vez que a exposição a ruídos em elevados índices de pressão sonora pode levar a perda auditiva induzida por ruído, qual o perfil audiológico de músicos com dedicação profissional? Tomou-se como base a perda auditiva como aquela induzida por ruído (PAIR) ou configurações audiométricas com entalhe que sugerem a PAIR. A busca de artigos ocorreu no período de 2 a 20 de junho de 2016, incluindo os seguintes bancos de dados eletrônicos: MEDLINE (via de acesso PubMed), Periódicos Capes e Biblioteca Virtual de Saúde (BVS).

Admitiram-se estudos observacionais datados a partir de 2006, realizada nos idiomas inglês, português e espanhol. Foram excluídos artigos de revisão e estudos de caso, ou estudos que estivessem associados à doenças preexistentes, alterações genéticas, síndromes, neuropatia auditiva e patologias da orelha média. 
A estratégia de busca utilizou os seguintes descritores de saúde (DeCS): "música", "perda auditiva induzida pelo ruído", "zumbido" e "audiometria" nas bases Periódicos Capes e BVS. Na base PubMed, utilizaram-se os termos MESH e seus correspondentes: "music", audiometry" e "hearing loss". As combinações entre as palavras-chave aconteceu em cada base de dados utilizando os operadores booleanos "e" e "and".

Além do idioma de publicação, utilizou-se como filtro na estratégia de pesquisa bibliográfica a presença dos descritores no título ou resumo. Uma vez finalizada a seleção dos artigos, procedeu-se à revisão das referências bibliográficas com o intuito de captar outras pesquisas não identificadas na pesquisa bibliográfica inicial.

Consideraram-se elegíveis aqueles artigos que, pela leitura do título e/ou do resumo, tinham como objetivo o estudo da perda auditiva em músicos. Os estudos identificados foram selecionados por dois avaliadores independentes, conforme conteúdo do título e resumo. Incluíram-se na revisão sistemática os artigos que estavam de acordo com os critérios estabelecidos. As discordâncias entre os avaliadores resolveram-se por consenso através da leitura da íntegra do estudo.

Construíram-se tabelas para apresentação dos principais resultados dos artigos selecionados, informações referentes aos autores, ano de publicação, país de realização, tamanho e descrição da amostra, modo de avaliação auditiva (testes auditivos), com o intuito de auxiliar na visualização na sessão resultados. Como estratégia de controle de viés, entrou-se em contato, via e-mail, com autores para consultar a existência de outros estudos realizados e ainda não publicados.

A avaliação da qualidade metodológica dos artigos selecionados foi realizada de acordo com os critérios estabelecidos pelas diretrizes STROBE (Strengthening the Reporting of Observational Studies in Epidemiology). Esse instrumento é constituído por 22 itens relacionados às informações que deveriam estar presentes no título, resumo, introdução, metodologia, resultados e discussão dos artigos. A Iniciativa STROBE foi elaborada por pesquisadores da área de epidemiologia, estatística e metodologia científica, e tem como objetivo disseminar os princípios que devem nortear a descrição de estudos observacionais. Decidiu-se avaliar qualitativamente com a classificação de "+", “-" e "?", de acordo com, respectivamente, a presença, ausência ou dados incompletos do critério estudado.

\section{RESULTADOS}

A estratégia de pesquisa bibliográfica permitiu identificar 819 artigos publicados no período entre 2006 e 2016. Após a leitura dos títulos, análise dos resumos e aplicação dos critérios de elegibilidade, incluíram-se 13 artigos à revisão sistemática. O número de artigos encontrados em cada base de dados de pesquisa está descrito na Figura 1.

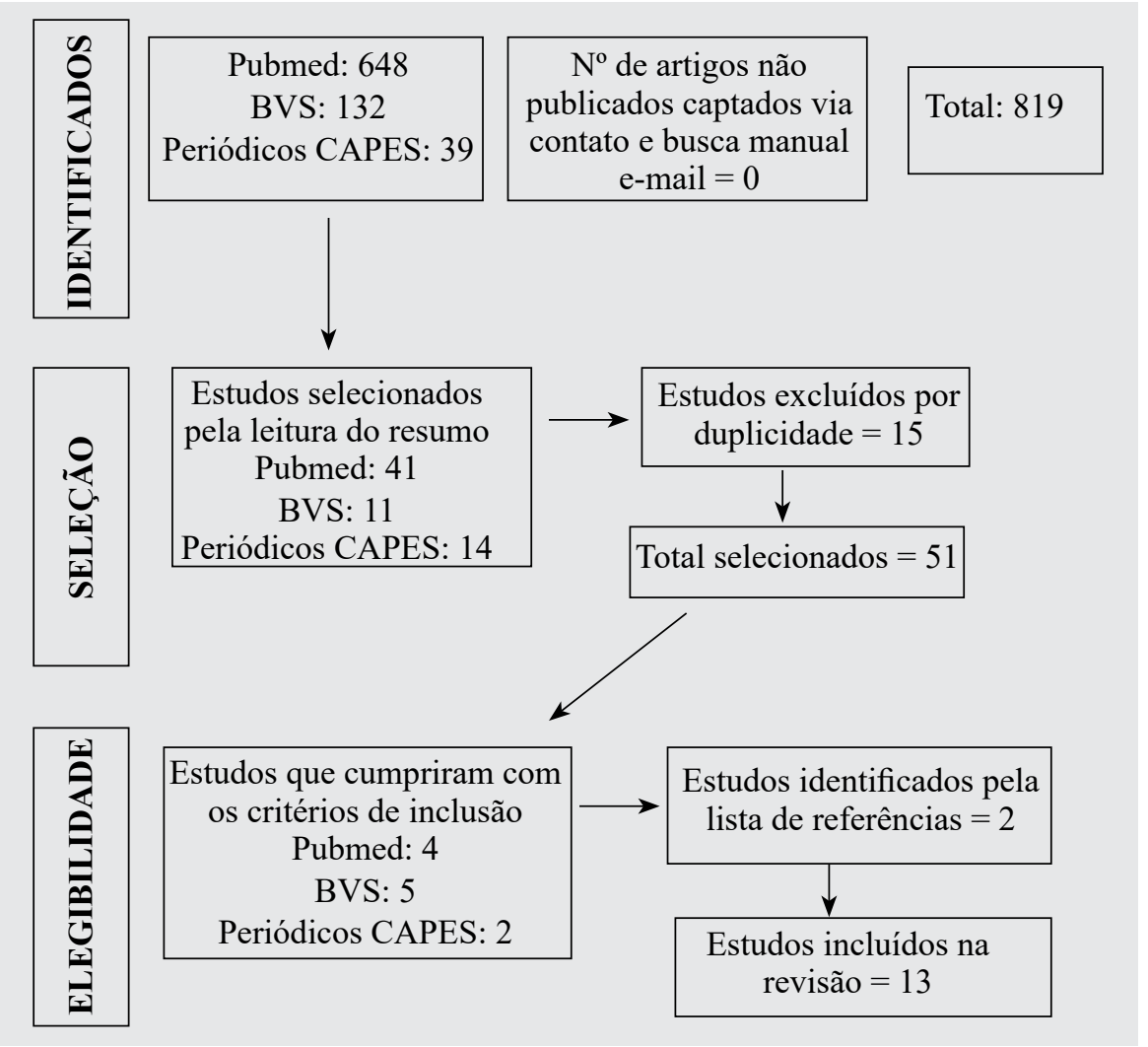

Figura 1 - Fluxograma de seleção dos artigos incluídos na revisão sistemática. $\mathrm{N}^{\mathrm{o}}$ : número. 
O Quadro I expressa os dados das características dos estudos referentes a autor, ano e país de publicação, campo musical, tamanho amostral e faixa etária da amostra. Aprecia-se que a maioria estudou adultos, de ambos os sexos, e na faixa etária entre 16 e 69 anos. Dois estudos avaliaram apenas homens ${ }^{(12,13)}$. Os estudos foram desenvolvidos em diferentes países, sendo $64,28 \%$ no Brasil e os demais em países europeus (Noruega, Suécia, Polônia, Inglaterra, Dinamarca e Finlândia). O tamanho amostral dos estudos era heterogêneo e variou entre 10 e 111 participantes. Quanto à faixa etária da amostra, esta não foi informada por 3 pesquisas $^{(14-16)}$ e uma delas indicou apenas a média de idade dos participantes ${ }^{(17)}$. Quanto ao campo musical, $14,28 \%$ eram músicos de pop rock/rock, 21,42\% músicos de orquestra, 21,42\% músicos de banda militar, 7,15\% ritmistas de escola de samba, 7,15\% violonistas e 28,58\% (cinco artigos) não informaram esse dado.

Quadro I - Características dos estudos incluídos: autor, ano e país de publicação, campo musical, tamanho da amostra e faixa etária.

\begin{tabular}{|c|c|c|c|c|}
\hline Autor & Ano & País & Campo musical & Tamanho amostra (faixa etária) \\
\hline Stormer et al.(7) & 2015 & Noruega & Músicos de Rock & 111 ( 16 e 52 anos) \\
\hline Gonçalves et al. ${ }^{(12)}$ & 2007 & Brasil & Banda Militar & 27 ( 22 e 50 anos) \\
\hline Samelli et al. ${ }^{(13)}$ & 2012 & Brasil & Não especificado & 16 (21 e 41 anos) \\
\hline Halevi-Katz et al. ${ }^{(14)}$ & 2015 & Suécia & Músicos pop/rock/jazz & 44 (não informada) \\
\hline Patil et al. ${ }^{(15)}$ & 2013 & Inglaterra & Músicos do exército & 84 (não informada) \\
\hline Toppila et al. ${ }^{(16)}$ & 2011 & Finlândia & Músicos de orquestra & 67 (não informada) \\
\hline Gonçalves et al. ${ }^{(17)}$ & 2009 & Brasil & Banda militar do exército & 50 (média de idade de 34,9 anos) \\
\hline Maia et al. ${ }^{(18)}$ & 2008 & Brasil & Não especificado & 23 ( 21 e 38 anos) \\
\hline Martins et al. ${ }^{(19)}$ & 2008 & Brasil & Não especificado & 21 (18 a 59 anos) \\
\hline Amorim et al. ${ }^{(20)}$ & 2008 & Brasil & Não especificado & 30 ( $18 \mathrm{~s}$ e 37 anos $)$ \\
\hline Monteiro et al. ${ }^{(21)}$ & 2010 & Brasil & Ritmistas de bateria de escola de samba & 10 (20 e 31 anos) \\
\hline Azevedo et al. ${ }^{(25)}$ & 2012 & Brasil & Violonistas & 10 (17 a 69 anos) \\
\hline Dudarewicz et al. ${ }^{(33)}$ & 2015 & Polônia & Músicos de orquestra & $18(30-58$ anos $)$ \\
\hline
\end{tabular}

No Quadro II apreciam-se os dados da prevalência da perda auditiva induzida por ruído (PAIR) e método de avaliação audiológica. Observa-se que cinco estudos não informaram dados sobre a prevalência de perda auditiva. Dos estudos que informaram dados de prevalência ${ }^{(7,12,13,15,17,19-21)}$, a soma dos participantes totalizou 349 , dos quais $31,24 \%$ apresentaram PAIR. Em relação aos exames audiológicos usados para avaliar a audição, a audiometria tonal aparece em todos os estudos, seguida da imitanciometria, 21,42\% das emissões otoacústicas evocadas por produto de distorção (EOAPD), 35,71\% emissões otoacústicas evocadas por transientes (EOAET), e audiometria vocal.

Apenas seis estudos informaram dados referentes a outros sintomas auditivos ${ }^{(7,17-19,21,25)}$. O zumbido foi o sintoma mais frequente e apareceu em todos os estudos. Outros sintomas citados foram dificuldade na compreensão da fala em ambiente ruidoso, intolerância a sons fortes ${ }^{(17,18)}$, plenitude auricular ${ }^{(18,19)}$, tontura $^{(17,19)}$ e otalgia ${ }^{(17)}$.

Quadro II - Dados referentes a prevalência de PAI R e método de avaliação audiológica.

\begin{tabular}{|c|c|c|}
\hline Autor & $\begin{array}{l}\text { Prevalência } \\
\text { de PAIR }\end{array}$ & Avaliação audiológica \\
\hline Stormer et al..$^{(7)}$ & $37,8 \%$ & Audiometria tonal, imitanciometria \\
\hline Gonçalves et al. ${ }^{(12)}$ & $18,5 \%$ & $\begin{array}{l}\text { Audiometria tonal, audiometria vocal, imitanciometria e teste do limiar de } \\
\text { desconforto sonoro }\end{array}$ \\
\hline Samelli et al. ${ }^{(13)}$ & $100 \%$ & Imitanciometria e audiometria tonal, EOAET e ABR e potencial cognitivo \\
\hline Halevi-Katz et al. ${ }^{(14)}$ & NI & Audiometria \\
\hline Patil et al..$^{(15)}$ & $14 \%$ & Audiometria tonal e vocal \\
\hline Toppila et al. ${ }^{(16)}$ & NI & Audiometria tonal \\
\hline Gonçalves et al. ${ }^{(17)}$ & $32 \%$ & Imitanciometria e audiometria tonal \\
\hline Maia et al..$^{(18)}$ & NI & Audiometria tonal, audiometria vocal, imitanciometria, EOAET e EOAPD \\
\hline Martins et al. ${ }^{(19)}$ & $42,9 \%$ & Audiometria \\
\hline Amorim et al. ${ }^{(20)}$ & $17 \%$ & Audiometria tonal, imitanciometria e EOAET e EOAPD \\
\hline Monteiro et al. ${ }^{(21)}$ & $40 \%$ & Audiometria tonal e vocal, imitanciometria \\
\hline Azevedo et al. ${ }^{(25)}$ & NI & Audiometria tonal, audiometria vocal, imitanciometria, EOAET e EOAPD \\
\hline Dudarewicz et al. ${ }^{(33)}$ & NI & EOAET \\
\hline
\end{tabular}

EOAET: emissões otoacústicas evocadas transites; EOAPD: emissões otoacústicas evocadas por produto de distorção; ABR: potenciais auditivos evocados; NI: não informado. 
Na intenção de recompilar dados sobre a vida laboral e o uso de equipamentos de proteção individual (EPI), dos 13 estudos, somente cinco continham dados sobre o tempo de profissão ${ }^{(12,17,19,20,25)}$ e apenas quatro ${ }^{(12,17,20,25)}$ apresentaram dados do tempo de exposição diária à música, cujos dados estão detalhados no Quadro III. Somente um estudo afirmou que $100 \%$ dos entrevistados utilizavam protetores auriculares como EPI. Os demais estudos não forneceram esse dado ${ }^{(21)}$.

Quadro III - Dados referentes ao tempo de profissão dos músicos e do tempo de exposição diária à música.

\begin{tabular}{|lll|}
\hline Autor & Tempo de profissão (anos) & Tempo de exposição diária a música (horas) \\
\hline Gonçalves et al. ${ }^{(12)}$ & Entre 5 e 26 anos & Entre 2 e 8 horas \\
Gonçalves et al. ${ }^{(17)}$ & $48 \%$ entre 11 e 20 anos & $44 \%$ de uma a dez horas por semana \\
& $26 \%$ entre 21 e 30 anos & \\
& $26 \% 1$ a 10 anos & Não informado \\
Martins et al. ${ }^{(19)}$ & $47,6 \% 6$ a 10 anos & \\
& $23,80 \% 11$ a 15 anos & \\
& $19 \% 16$ a 20 anos & $43,3 \%$ de 1 a 4 horas \\
Amorim et al. ${ }^{(20)}$ & $53,33 \%$ até 10 anos & $36,6 \%$ entre 4 e 7 horas \\
& $46,66 \%$ mais de 10 anos & $20 \%$ entre 7 e 10 horas \\
Azevedo et al. ${ }^{(25)}$ & $9,5 \%$ maior que 20 anos & Média semanal 15,2 horas \\
\hline
\end{tabular}

Com base no Quadro IV, a avaliação da qualidade metodológica dos estudos incluídos foi avaliada. No que se refere a esse item, os critérios determinados pelo STROBE foram seguidos. Nenhum estudo informou dados em relação aos itens 16, 17 e 22 (respectivamente estimativas dos resultados, análises extras e fontes de financiamento). Todos os estudos responderam de forma incompleta ao item 20 (interpretação dos resultados); e os itens 5, 7 e 11 foram os mais inconsistentes.

Quadro IV - Qualidade dos estudos segundo os critérios do STROBE (Strengthening the Reporting of Observational Studies in Epidemiology).

\begin{tabular}{|c|c|c|c|c|c|c|c|c|c|c|c|c|c|c|c|c|c|c|c|c|c|c|}
\hline Autores/itens & 1 & 2 & 3 & 4 & 5 & 6 & 7 & 8 & 9 & 10 & 11 & 12 & 13 & 14 & 15 & 16 & 17 & 18 & 19 & 20 & 21 & 22 \\
\hline Stormer et al. ${ }^{(7)}$ & + & + & - & + & + & + & $?$ & + & + & + & $?$ & $?$ & + & + & + & - & - & + & + & $?$ & + & - \\
\hline Gonçalves et al. ${ }^{(12)}$ & - & - & - & - & $?$ & $?$ & $?$ & + & + & + & + & $?$ & + & + & + & - & - & + & - & $?$ & - & - \\
\hline Samelli et al. ${ }^{(13)}$ & + & + & - & + & $?$ & + & $?$ & + & $?$ & + & + & - & + & + & + & - & - & + & - & $?$ & + & - \\
\hline Halevi-Katz et al. ${ }^{(14)}$ & - & + & + & + & $?$ & + & $?$ & $?$ & $?$ & + & - & - & + & + & + & - & - & + & - & $?$ & + & - \\
\hline Patil et al. ${ }^{(15)}$ & - & + & - & + & $?$ & + & $?$ & + & - & + & + & - & + & + & + & - & - & + & - & $?$ & + & - \\
\hline Toppila et al. ${ }^{(16)}$ & - & - & $?$ & + & - & + & $?$ & + & $?$ & + & + & $?$ & + & + & + & - & - & + & - & $?$ & + & - \\
\hline Gonçalves et al. ${ }^{(17)}$ & - & - & - & + & $?$ & + & $?$ & + & - & + & $?$ & - & + & + & + & - & - & + & - & $?$ & + & - \\
\hline Maia et al. ${ }^{(18)}$ & - & - & $?$ & + & $?$ & + & + & + & + & + & + & $?$ & + & + & $?$ & - & - & + & - & $?$ & - & - \\
\hline Martins et al. (19) & + & + & - & + & $?$ & + & $?$ & + & + & + & - & - & + & + & + & - & - & + & - & $?$ & - & - \\
\hline Amorim et al. ${ }^{(20)}$ & + & + & - & + & $?$ & + & - & + & + & + & + & + & + & + & + & - & - & + & - & $?$ & - & - \\
\hline Monteiro et al. ${ }^{(21)}$ & + & - & - & + & $?$ & + & $?$ & + & + & + & $?$ & - & + & + & + & - & - & + & - & $?$ & - & - \\
\hline Azevedo et al. ${ }^{(25)}$ & - & $?$ & - & + & + & + & $?$ & + & - & + & + & - & + & + & + & - & - & + & - & $?$ & - & - \\
\hline Dudarewicz et al. ${ }^{(33)}$ & - & + & - & + & + & + & $?$ & + & $?$ & + & $?$ & - & + & + & + & - & - & + & - & $?$ & - & - \\
\hline
\end{tabular}

“+” dado presente, “-“"dado ausente e "??" dado incompleto

1-Titulo e resumo; 2-Contexto e justificativa; 3-objetivos; 4-Desenho do estudo; 5-Contexto;6-Métodos/Participantes; 7-Variaveis; 8-Fonte de dados/mensuração; 9-Viés; 10-Tamanho do estudo; 11-Variaveis quantitativas;12-Métodos estatísticos; 13-Resultsados/Participantes; 14-Dados descritivos; 15-Desfecho; 16- Resultados principais; 17-Outras análises; 18-Discussão/Resultados; 19-Apresentação das Limitações; 20-Interpretação dos resultados; 21-Generalizações dos resultados; 22-Financiamento.

\section{DISCUSSÃO}

Os profissionais da música nem sempre são vistos como população de risco para desenvolvimento de PAIR, pois ela, corriqueiramente, está associada a momentos de lazer. No entanto, a música gera ruídos e, em consequência, eles poderão causar prejuízos auditivos permanentes ${ }^{(22,23)}$. $\mathrm{O}$ aumento na potência dos amplificadores acoplados aos instrumentos musicais modernos podem potencializar essa problemática.

Os achados dessa revisão permitiram observar que a perda auditiva induzida por ruído é frequente em músicos, o que salienta a importância de estudos sobre a saúde auditiva nessa população, tanto no que se refere à ocorrência de sinais e sintomas auditivos quanto ao uso de equipamentos de proteção individual. A identificação de 13 artigos que atendiam aos critérios 
de inclusão demonstra que a avaliação auditiva dos profissionais da música é uma questão pouco discutida na comunidade científica.

Pesquisadores na Alemanha, entre os anos de 2004 a 2008, compararam a incidência de perda auditiva entre 2.227 músicos e a população em geral, e expuseram que os músicos apresentaram 3,51 vezes mais risco de desenvolver PAIR e 1,45 vezes mais zumbido do que a população em geral ${ }^{(24)}$. Outra pesquisa demonstrou que o aumento de perdas auditivas em músicos é esperado, sobretudo pela modernização dos equipamentos sonoros, geradores de sons mais potentes, o que expõe os mesmos a níveis de pressão sonora mais elevados e, nesse sentido, a prevalência de PAIR tem sido encontrada semelhante ao achado em trabalhadores de indústrias metalúrgicas ${ }^{(10)}$. A presença do entalhe audiométrico, mesmo em uma única frequência, deveria ser encarada como um sinal de alerta, pois sugere tendência ao desencadeamento da perda auditiva pela exposição a níveis de pressão sonora elevados com o decorrer do tempo ${ }^{(10)}$.

A falta de regulamentação dessa profissão torna omisso o estabelecimento de critérios que preservem a saúde desse trabalhador: determinação da jornada de trabalho, obrigatoriedade de equipamentos de proteção individual, estabelecimentos de exames periódicos de verificação da saúde auditiva ${ }^{(8,10)}$. De acordo com alguns autores ${ }^{(7,12,14,16,20,21,25)}$, o potencial risco de PAIR existe em diferentes campos musicais (rock, trios elétricos, orquestras sinfônicas, escolas de samba); não só no uso coletivo de instrumentos musicais, mas também no uso individual desses. O estudo de fatores de risco para PAIR deve levar em consideração as fontes sonoras do ruído, o tempo de permanência no ambiente ruidoso e o uso de equipamentos de proteção.

Observou-se que a maioria dos participantes estudados referiu zumbido, sintoma auditivo que pode indicar perda auditiva, porém precisa ser demonstrado por exames audiométricos. Quando o corpo é exposto a níveis elevados de pressão sonora, ele reage a esse estímulo, e tais reações poderão se tornar permanentes e criar alterações orgânicas e psicológicas ${ }^{(26)}$. $\mathrm{O}$ impacto do ruído pode perturbar o trabalho, o descanso, o sono e a comunicação em seres humanos. Um estudo sobre audição, zumbido e qualidade de vida evidenciou que o zumbido interferia no estado emocional, impactava na qualidade do sono, concentração e na vida social ${ }^{(27)}$. Além dos sintomas relacionados à audição, muitos profissionais relataram dificuldade de compreensão da fala em ambiente ruidoso, intolerância a sons fortes, plenitude auricular, otalgia e tontura, o que permite afirmar que a exposição contínua ao ruído também resulta no aparecimento de sintomas extra-auditivos que afetam o bem-estar e, consequentemente, a qualidade de vida do músico ${ }^{(28)}$.

Ao estudar as implicações sociais da deficiência auditiva adquirida em adultos, a dificuldade de compreensão da fala enseja a dificuldade de comunicação no grupo social. Relatos da percepção dos familiares são significativos sobre as dificuldades enfrentadas no meio social, e contribuem para o entendimento da exclusão vivenciada pelas pessoas que têm perda auditiva, isto é, questões como alteração de comportamento e irritação. A vergonha de pedir às pessoas que repitam o que falaram, ou que falem mais alto, faz com que sejam vítimas de zombaria, contribuindo para o isolamento e o desenvolvimento de situações de estresse, cobrança de si próprio e ansiedade ${ }^{(2)}$.

Evidenciou-se nos estudos uma falta de clareza e informações quanto ao uso do EPI pelos músicos, o que sugere uma discussão mais aprofundada sobre a necessidade da percepção individual dos ruídos no ambiente de trabalho e dos riscos a que esses profissionais estão expostos, principalmente porque, muitas vezes, eles banalizam os riscos ocupacionais e não sabem identificar as consequências decorrentes da inobservância do uso de medidas de prevenção. Assim, recomenda-se que mais do que realizar educação continuada sobre a audição ou sobre o uso de equipamentos de proteção auditiva, é imprescindível criarse e implementar-se um Programa de Prevenção de Perdas Auditivas (PPPA), que deve ter como meta provocar mudanças de comportamento $^{(6,29,30)}$.

Identificaram-se carências metodológicas nos estudos selecionados e sobressaíram-se os itens 1, 3, 5, 7, 12, 17, 19, 20 e 21 da Iniciativa STROBE, referentes às questões, respectivamente, sobre estrutura do resumo; explanação dos objetivos; contexto em que o estudo foi realizado; detalhamento das variáveis de estudo; métodos estatísticos aplicados; realização de análises extra; apresentação das limitações do estudo; interpretação dos resultados; generalizações dos resultados.

A falta de informação em relação à metodologia empregada nos estudos avaliados na presente revisão traz limitações para a discussão mais abrangente dos resultados, o que dificulta comparações. Nos estudos incluídos, a metodologia apresentada não trouxe informações suficientes para que os resultados obtidos pudessem ser comparados entre si.

Cabe destacar que o método científico é o caminho para a construção do conhecimento e para a reprodutibilidade de um estudo, desde que descrito adequadamente ${ }^{(31,32)}$. A escolha e a descrição do método utilizado com detalhamento de dados possibilitam um planejamento eficaz do estudo e permitem a averiguação por outros pesquisadores. A Iniciativa STROBE disponibiliza a lista para verificação dos itens que devem estar presentes em estudos observacionais, contribuindo para a maior fidedignidade das pesquisas e para a reprodutibilidade dos estudos.

Nesta revisão, não foi possível relacionar a perda auditiva ao tempo de exposição diária devido à falta de dados apresentados nos estudos originais. As interpretações acerca do uso de equipamento de proteção individual, como instrumento de proteção auditiva, são limitadas, visto que, na maioria dos estudos, esse tema não foi investigado. A qualidade metodológica dos estudos inibe a possibilidade de elucidar os efeitos do ruído na audição de músicos, principalmente no que se refere aos níveis de pressão sonora e tempo de exposição. 


\section{CONCLUSÃO}

Por meio desta revisão foi possível identificar que os achados da literatura são consensuais quanto à detecção de que músicos apresentam perda auditiva induzida por ruído, sendo o zumbido o sintoma auditivo mais frequente, fenômeno indicativo de que existe alteração na audição desses profissionais. $\mathrm{O}$ uso de equipamento de proteção individual, como protetores auriculares, parece não ser rotina entre a população estudada.

\section{AGRADECIMENTOS}

Ao financiamento da Fundação de Amparo à Pesquisa e ao Desenvolvimento Científico e Tecnológico do Maranhão (FAPEMA).

\section{CONFLITOS DE INTERESSE}

Autores declaram não haver conflitos de interesses.

O presente estudo compõe parte da dissertação intitulada “Audição na toada de Boi”, apresentada à Universidade CEUMA em 2017, 127 páginas.

\section{REFERÊNCIAS}

1. Tung CY, Chao KP. Effect of recreational noise exposure on hearing impairment among teenage students. Res Dev Disabil. 2013;34(1):126-32.

2. Francelin MAS, Motti TFG, Morita I. As Implicações sociais da deficiência auditiva adquirida em adultos. Saúde Soc. 2010;19(1):180-92.

3. World Health Organization. Deafness and hearing loss; Fact Sheet No 300, Updated March 2015 [acesso em 2016 Dez 20]. Disponível em: www.who.int/entity/mediacentre/factsheets/fs300/en/

4. Instituto Brasileiro de Geografia e Estatística - IBGE. Ciclos de vida: Brasil e grandes regiões. 2013 [acesso em 2016 Nov 25]. Disponível em: http://biblioteca.ibge.gov.br/visualizacao/livros/liv94522.pdf

5. Vieira EMA. Risco ocupacional relacionado ao conforto ambiental em UTIs. [dissertação]. João Pessoa: Universidade Federal da Paraíba; 2016.

6. Munhoz G, Lopes A. Programa de Prevenção de Perdas Auditivas (PPPA) para Músicos. Rev Portuguesa Saúde Ocupacional [Internet]. 2016. [acesso em 2016 Nov 02]. Disponível em: http://www.rpso.pt/programa-de-prevencao-deperdas-auditivas-pppa-para-musicos/

7. Stormer CC, Laukli E, Høydal EH, Stenklev NC. Hearing loss and tinnitus in rock musicians: a Norwegian survey. Noise Health. 2015; 17(179):411-21.

8. Luders D, Gonçalves CGO. Trabalho e saúde na profissão de músico: reflexões sobre um artista trabalhador. Tuiuti Ciência Cultura. 2013;(47):123-37.

9. Ottoni AO, Barbosa-Branco A, Boger ME, Garavelli SL. Study of the noise spectrum on high frequency thresholds in workers exposed to noise. Rev Bras Otorrinolaringol. 2012;78(4):108-14.

10. Regis ACFC, Crispim KGM, Ferreira APF. Incidência e prevalência de perda auditiva induzida por ruído em trabalhadores de uma indústria metalúrgica, Manaus-AM, Brasil. Rev CEFAC. 2014;16(5):1456-62.

11. Moher D, Liberati A, Tetzlaff J, Altman DG, PRISMA Group. Reprint-preferred reporting items for systematic reviews and meta-analyses: the PRISMA statement. Phys Ther. 2009;89(9):873-80.

12. Gonçalves MS, Tochetto TM, Gambini C. Hiperacusia em músicos de banda militar. Rev Soc Bras Fonoaudiol. 2007; 12(4):298-303.

13. Samelli AG, Matas CG, Carvallo RM, Gomes RF, Beija CS, Magliaro FC, et al. Audiological and electrophysiological assessment of professional pop/rock musicians. Noise Health. 2012;14:6-12.

14. Halevi-Katz DN, Yaakobi E, Putter-Katz H. Exposure to music and noise-induced hearing loss (NIHL) among professional pop/rock/jazz musicians. Noise Health. 2015;17(76):158-64.

15. Patil ML, Sadhra S, Taylor C, Folkes SEF. Hearing loss in British Army musicians. Occup Med. 2013;63(4):281-3. 
16. Toppila E, Koskinen H, Pyykkö I. Hearing loss among classical-orchestra musicians. Noise Health. 2011; 13(50):45-50.

17. Gonçalves CGO, Lacerda ABM, Zocoli AMF, Oliva FC, Almeida SB, Iantas MR. Percepção e o impacto da música na audição de integrantes de banda militar. Rev Soc Bras Fonoaudiol. 2009;14(3):515-20.

18. Maia JRF, Russo ICP. Estudo da audição de músicos de rock and roll. Pró-Fono R Atual Cient. 2008;20(1):49-54.

19. Martins JPF, Magalhães MC, Sakae TM, Magajewski FRL. Avaliação da perda auditiva induzida por ruído em músicos de Tubarão-SC. Arq Catarin Med. 2008;37(4):69-74.

20. Amorim RB, Lopes AC, Santos KTP, Melo ADP, Lauris JRP. Auditory Alterations for Occupational Exposition in Musicians. Int Arch Otorhinolaryngol. 2008;12(3):377-83.

21. Monteiro VM, Samelli AG. Estudo da audição de ritmistas de uma escola de samba de São Paulo. Rev Soc Bras Fonoaudiol. 2010;15(1):14-8.

22. Barcelos DD, Dazzi NS. Efeitos do mp3 player na audição. Rev CEFAC. 2014;16(3):779-91.

23. Rodrigues MA, Freitas MA, Neves MP, Silva MV. Evaluation of the noise exposure of symphonic orchestra musicians. Noise Health. 2014;16(68):40-6.

24. Schink Tania, Kreutz G, Busch V, Pigeot I, Ahrens W. Incidence and relative risk of hearing disorders in professional musicians. Occup Environ Med. 2014;71(7):472-6.

25. Azevedo MF, Oliveira C. Audição de violinistas profissionais: estudo da função coclear e da simetria auditiva. Rev Soc Bras Fonoaudiol. 2012;17(1):73-77.

26. Khan N, Khan AR, Khan A, Khan S. Frequência de perda de audição em ambientes ocupacionais ruidosas. Rev Ciências Médicas. 2012;20(4):192-5.

27. Esteves CC, Brandão FN, Siqueira CGA, Carvalho SAS. Audição, zumbido e qualidade de vida: um estudo piloto. Rev CEFAC. 2012;14(5):836-43.

28. Oliveira RCO, Santos JS, Rabelo ATV, Magalhães MC. O impacto da exposição ao ruído nos trabalhadores em Unidades de suporte móvel. CODAS. 2015;27(3):215-22.

29. Olson AD, Gooding LF, Shikoh F, Graf J. Hearing Health in College Instrumental Musicians and Prevention of Hearing Loss. Med Probl Perform Art. 2016;31(1):29-36.

30. Lüders D, Gonçalves CGO, Lacerda ABM, Schettini SRL, Silva LSG, Albizu EJ, et al. Audição e qualidade de vida de músicos de uma orquestra sinfônica brasileira Audiol Commun Res. 2016;21:e1688.

31. Amatuzzi MLL, Amatuzzi MM, Leme LEG. Metodologia científica: o desenho da pesquisa. Acta Ortop Bras. 2003;11(1 Supl 1):58-61.

32. Trindade DB, Esquivel RM, Amorim LDAF. Tamanho amostral para análise de medidas repetidas em estudos longitudinais. In: Simpósio Nacional de Probabilidade e Estatística, 2011; São Pedro, São Paulo. São Paulo: Associação Brasileira de Estatística; 2011.

33. Dudarewicz MPŁ, Małgorzata ZD, Kamil Z. Exposure to excessive sounds during orchestra Rehearsals and temporary hearing changes in hearing among musicians. Med Pr. 2015;66(4):479-86.

\section{Endereço do primeiro autor:}

Carina Moreno Dias Carneiro Muniz

Universidade CEUMA

Rua Josué Montelo, 1

Bairro: Renascença 2

CEP: 65075-120 - São Luis - MA - Brasil

E-mail: carina.muniz@ifma.edu.br

\section{Endereço para correspondência:}

Rosane da Silva Dias

Universidade CEUMA

Rua Josué Montelo, 1

Bairro: Renascença 2

CEP: 65075-120 - São Luis - MA - Brasil

E-mail: rosanesdias@hotmail.com 